\title{
THE KIRKWOOD GAPS AS AN ASTEROIDAL SOURCE OF METEORITES
}

\author{
H. SCHOLL and C. FROESCHLE
}

Zimmerman and Wetherill's proposed mechanism for the production of meteorites from asteroids has been explored by numerous numerical computations of orbits. Starting from the vicinity of the $2 / 1 \mathrm{Kirkwood} g a p, 30 \%$ of our orbits soon have aphelia reaching beyond, but not much beyond 4 A.U.; ( $3 \%$ only exceed 4.3 A.U.). These results suggest that the proposed mechanism might work, although we have not yet checked whether Jupiter's action will be large enough for those perihelia. Furthermore, our investigation has been extended to the $3 / 1,7 / 3$ and $5 / 2$ Kirkwood gaps, and shows that these gaps should also be considered as a possible source of meteorites.

Certain classes of meteorites are presumed to originate in the asteroidal helt between the orbits of Mars and Jupiter. These meteorites are considered to be fragments resulting from collisions between asteroids that have perihelia outside the orbit of Mars. The orbits of the fragments are changed into earthcrossing orbits by some specific mechanism, and after a collision with the Earth, the fragments appear as meteorites on the Earth.

The dynamical problem is to find such a mechanism that transforms non earth-crossing orhits into earth-crossing ones. Direct collisional transfer into earth-crossing orbits gererally is excluded as it requires a strong change in velocity which should produce shock cffects in the meteorites that are not observed (wotheril1 1974). 'Therefore, other mechanisms have to be found. As is wellknown, there are gaps in the frequency distribution of the asteroids' mean motions, the so-called kirkwood gaps, where the ratio between the asteroids' and Jupiter's mean motion is close to a small rational number. If an asteroid is $10-$ cated in one of commensurability gaps it will he in resonant motion with. Jupiter. P. D. Zimmerman and (i. W. Wetherill (1973) proposed a mechanism that yields carth-crossing orbits from the Kirkwood gap which is at the $2 / 1$ commensurability in the asteroidal bolt.

The orbit of a fictitious asteroid with normal starting values placed at the $2 / 1$ commensurability shows much stronger perturbations than an asteroid that is not in a resonant motion with Jupiter. Particularly, the eccentricity of the orbit varies strongly while the semimajor axis remains nearly constant according to Poisson's theorem. Subsequently, the aphelion of the orbit may approach closely Jupiter's orbit. Zimmerman and Wetherill hase their proposed mechanism for the yield of meteorites on that effect. Two asteroids, which are close to the $2 / 1$ commensurability, collide and hreak up. A fragment enters the gap and, according to the mechanism described above, approaches closely Jupiter's orbit. 
While in resonant motion, elose approaches to Jupiter itself will not occur. Eventually a statistically probable second collision will remove fragments from resonance and close approaches not only to Jupiter's orbit, hut to Jupiter itself become frequent. Then, Jupiter's perturbations may change the fragment's orbit into an earth-crossing orbit.

There remain two problems: What starting values for the fragment in the gap yield sufficiently strong variations in eccentricity and what is the limiting value for the aphelia in order to transform orbits into earth-crossing orbits hy perturbations of Jupiter?

We investigated the regions near the $2 / 1$ commensurahility for strong variations in eccentricity hy numerical calculations. Our model was hased on the plane elliptic restricted three body problem averaged hy Schubart's method (Schuhart 1964). In that model, an orbit is determined hy four quantities a, e. $\sigma, \mu$; a is the semimajor axis, $O$ is the eccentricity of the fragment's orhit. $\mu$ and $\sigma$ are the angles which determine the relative position hetween Jupiter and the fragment as well as the angle between their peribelia. The starting values for an orbit were chosen in the a, ${ }^{\prime}$. n phase space defined by 3.25 a 5.3 .36 All. $0^{\circ} \leq \pi \leq 360^{\circ}, 0 \leq e \leq 0.14$. The limits for a correspond to the ohserved limits of the gap. For further details regarding the calculations see scinool and Froeschlé (1974, 1975).

We calculated about 100 orbits numerically over 10,000 years to find the frequency distribution of the aphelia. According to our results, about $30 \%$ of the orbits starting in the phase space defined above have aphelia beyond 4 All. However, after 4 AU there is a sharp drop-off in the frequency distribution curve. Only $8 \%$ of the orbits have aphelia larger than $4.1 \mathrm{All}$ and only $3 \%$ exceed $4.3 \mathrm{All}$. No orbit was found with an aphelion larger than 4.5 All. No significant number of orbits approaches Jupiter's orbit within 0.05 AlJ since Jupiter's perihelion is at about $5 \mathrm{AU}$.

We have not yet checked whether Jupiter's action will he large enough to transform these orhits into earth-crossing ones. If Jupiter's action extends down to $4 \mathrm{AU}$ or less, our results support well their proposed mechanism.

Furthermore, we investigated orhits at three other Kirkwood gaps, (the 5/2, $7 / 3$ and $3 / 1$ commensurabilities) for close approaches with Jupiter's orbit. For the $7 / 3$ case, no orbit had an aphelion larger than 4 AU. Therefore, the $7 / 3$ case is excluded as a possible source for meteorites. In the $5 / 2$ gap, a surprisingly large $30 \%$ of the orbits exceeded 4 AU. The phase space for the starting values was chosen as for the $2 / 1$ case. We are not able to state how many orbits exceed 4.1 AU or 4.3 AU because at the $5 / 2$ commensurability; the eccentricity varies with long periods - more than 50,000 years in several cases. As the computing time was 1 imited, we usually stopped the calculations when an orbit exceeded $4 \mathrm{AU}$ which was equivalent to a value of 0.41 for the eccentricity. Some selected orbits were integrated over longer periods of time. They yielded aphelia at 4.5 AU. Therefore, the Kirkwood gap at the $5 / 2$ commensurability also can be regarded as a source for meteorites.

No orbit starting in the Kirkwood gap at the $3 / 1$ commensurability has an aphelion close to $4 \mathrm{AU}$. That would require an ecrentricity of 0.6 . However, because of the variations in eccentricity, the perihelia of some orbits approach closely the orbit of Mars. $26 \%$ of the orbits have perihelia less than 1.9 AU, $15 \%$ are below $1.8 \mathrm{AU}$ and $6 \%$ come below $1.7 \mathrm{AU}$. The aphelion of the orbit of Mars is $1.66 \mathrm{AU}$. There remains the problem of determining the zone around Mars in which Mars is able to transform these particular orbits into earth-crossing orbits.

In conclusion, we can say that in addition to the Kirkwood gap at the $2 / 1$ commensurability proposed by Zimmerman and Wetherill, the Kirkwood gaps at the $5 / 2$ and at the $3 / 1$ commensurability also may be considered as possibje sources for meteorites. However, we must say, that all nur calculations are based on the planar restricted three body problem and therefore, calculations hased on 


\section{METEORITES FROM THE KIRKWOOD GAP}

a three dimensional model might yield different results. The coupling hetween the variation in eccentricity and between the motion of the argument in perihelion of a fragment's orbit may prevent close approaches with Jupiter or with Mars.

\section{ACKNOWLEDGEMENTS}

We thank Dr. J. Schubart for his continuing interest. The calculations were carried out on the two IBM $370 / 168$ of the Iniversity of Heidelherg and of the C.I.R.C.E. at Orsay respectively.

\section{REFERENCES}

Schol1, H., and Freesthle. C. 1974, Astron. and Astrophys., 33, 455. Scholl, H., and Frowschli, (. 1975. Astron. and Astrophys. 42. 457. Schubart, J. 1964, SAO Special Report No. 149.

Wetherill, G. W. 1974, in Ann. Rev. of Earth and Pl. Sciences, Vol. 2. 303. Zimmerman, P. D., and Wetherill, G. W. 1973, Science, 182, 51. 